\title{
Reseña: "Terapia afirmativa: una introducción a la psicología y a la psicoterapia dirigida a gays, lesbianas y bisexuales"
}

\section{Review: "Affirmative therapy: an introduction to psychology and psychotherapy aimed to gays, lesbians and bissexuais"}

\section{Revisão: "Terapia afirmativa: uma introdução à psicologia e psicoterapia visando gays, lésbicas e bissexuais"}

Borges, K. (2009). Terapia afirmativa: uma introdução à psicologia e à psicoterapia dirigida a gays, lésbicas e bissexuais. São Paulo: Edições GLS.

Paribanú Freitas De León Universidad de la República, Uruguay

Autor referente: paribanufreitas@psico.edu.uy

Historia Editorial:

Recibido: 09/07/2017

Aceptado: 19/02/2018

\section{RESUMEN}

Se presenta el libro "Terapia afirmativa: uma introdução à psicologia e psicoterapia visando gays, lésbicas e bissexuais" del psicólogo brasileño Klecius Borges, publicado en el año 2009. El mismo tiene relevancia sustantiva para el desarrollo académico y profesional en Psicología ya que es una de los pocos aportes introductorios al trabajo con personas LGBTI desde un enfoque afirmativo. Se presentan los principales tópicos analizados por el autor y sus aportes.

Palabras clave: Terapias afirmativas; Estudios LGBT; Diversidad sexual; Psicología 


\section{ABSTRACT}

The article revies the book "Affirmative therapy: an introdução à psicologia e psicoterapia visando gays, lésbicas e bissexuais" written by the brazilian psychologist Klecius Borges in 2009. It has substantive relevance for academic and professional development in
Psychology because it is one of the few introductory contributions to work with LGBTI people from an affirmative approach. The article presented the main topics analyzed by the author and their contributions.

Keywords: Affirmative therapies; LGBT studies; Sexual diversity; Psychology

\section{RESUMO}

É apresentado o livro "Terapia afirmativa: uma introdução à psicologia e psicoterapia visando gays, lésbicas e bissexuais" do psicólogo brasileiro Klecius Borges, publicado em 2009. O livro tem relevância substancial para o desenvolvimento acadêmico e profissional em Psicologia, já que é um das poucas contribuições introdutórias para trabalhar com pessoas LGBTI de uma abordagem afirmativa. No artigo apresentam-se principais tópicos analisados pelo autor e suas contribuições são apresentados.

Palavras chave: Terapias afirmativas; Estudos LGBT; Diversidade sexual; Psicologia

\section{Pertinencia}

$\mathrm{S}$ i bien como el propio autor indica, el término Terapias Afirmativas (originalmente "Affirmative therapy" en inglés), fue acuñado en 1982, su uso y difusión en la psicología iberoamericana es particularmente escasa.

De hecho, una revisión de este término en bases de datos de revistas científicas con acceso abierto tales como Scielo o Redalyc, darán como resultado la existencia de un único artículo; el titulado "Terapia afirmativa para homosexuales y lesbianas", publicado en la "Revista colombiana de psiquiatría" por Ardila (2007). La situación del pobre abordaje específico del campo se torna lastimosa cuando lo comparamos con el nada pobre nivel de desarrollo del tema a nivel anglosajón.

De hecho, una búsqueda básica pero estricta realizada a los efectos de esta reseña en el portal TIMBÓ, patrocinado por la Agencia Nacional de Investigación e Innovación 
del Uruguay, arroja la existencia la existencia de más de 280 artículos arbitrados específicos, escritos en inglés.

De allí que el libro del psicólogo brasileño Klecius Borges adquiere una relevancia fundamental con su sola existencia; al tornar visible a la comunidad profesional íberohablante un área de desarrollo de la psicología, absolutamente ignorado en la región.

Más allá de este gesto, el texto de Borges tiene además la virtud de funcionar como una excelente introducción a los temas específicos de las Terapias Afirmativas, así como también a sus principales autores (Cass, 1979; Coyle \& Kitzinger, 2002; Davies \& Neal, 1996; Kort, 2008; Neal \& Davies, 2000).

Conocimientos a los que se suma su experiencia clínica; la cual emerge de la atención sistemática a pacientes lesbianas, gays, bisexuales y transgénero a lo largo de una década.

\section{Estructura}

Página a página, la lectura del libro hace viajar al lector por diferentes tópicos vinculados a la relación entre psicología y estudios LGBTIQ (Lésbico - Gay - Bi Trans - Intersex y Queer). Tópicos estos que puede haber pensado espontáneamente en el marco de las actuales agendas progresistas y de derechos; pero tópicos también que seguramente resulten de absoluta novedad, incluso para profesionales relativamente familiarizados con las comunidades y culturas LGBTIQ.

Estructurado en siete capítulos, el autor recorre un camino que va de lo general a lo particular.

En los dos primeros capítulos, Borges aborda la definición, historia, necesidad y fundamentos de lo que denomina una "Psicología homosexual" (Capítulo 1); 
principalmente para ponerlo en diálogo con la especificidad de las Terapias Afirmativas (Capítulo 2).

En relación con esto, Borges es meridianamente claro al señalar la necesidad de desarrollar una "Psicología homosexual" como piedra angular de los enfoques afirmativos en psicoterapia; recordando que

La llamada psicología homosexual (lesbian and gay psychology) surgió en los Estados Unidos y se difundió en países europeos, principalmente en Gran Bretaña, en reacción al heterocentrismo de la psicología dominante - que, hasta mediados de los años 1970, continuaba presentando la homosexualidad como una manifestación patológica, y a los homosexuales como producto de un desarrollo psicosexual problemático (Borges, 2009, Capítulo 1, Posición 82) [Traducción del autor].

Esta reacción permitió un giro sustantivo en la forma de mirar a las personas LGBTI desde la disciplina, y propició el desarrollo de conocimiento en profundidad sobre una variedad de temas de interés específico para dicha población, tales como el establecimiento de las identidades, lésbica, gay y bisexual; la formación de relaciones entre personas del mismo sexo, los procesos de desarrollo de la sexualidad, la homofobia y la discriminación sufrida por gays y lesbianas; las cuestiones relativas a la maternidad y paternidad; la diversidad cultural y étnica entre lesbianas, gays y bisexuales; [0] las cuestiones sobre las elecciones y las prácticas sexuales (Borges, 2009, Capítulo 1, Posición 108) [Traducción del autor].

Recordando que el estudio de estos tópicos permitió la elaboración de "un conjunto de presupuestos teóricos sobre la homosexualidad y una actitud clínica específicamente orientada al desarrollo de una identidad homosexual positiva" (Borges, 2009, Capítulo 
Introducción, Posición 71) [Traducción del autor] que define a las Terapias Afirmativas. Destacando además que:

1. A nivel teórico

la terapia afirmativa no ve al sujeto homosexual como psicológicamente diferente del sujeto heterosexual. Sin embargo, al segmentar las orientaciones sexuales y definir un foco, ella busca una comprensión más profunda de las cuestiones particulares de esa población, así como el desarrollo de modelos teóricos y clínicos más adecuados (Borges, 2009, Capítulo 1, Posición 108) [Traducción del autor].

2. Que ni la Psicología Homosexual - que como bien señala el autor, en la actualidad incorpora en su consideración a la población bisexual y transgénero - , ni las Terapias Afirmativas se pueden centrar o reducir a "una psicología de la sexualidad, pues no se restringe a [la consideración de] las prácticas sexuales" (Borges, 2009, Capítulo 1, Posición 108) [Traducción del autor].

Un punto importante, además, a considerar por el lector, es que lo que se nomina como "Terapias Afirmativas", no constituyen un sistema teórico - técnico radicalmente independiente respecto de los sistemas psicoterapéuticos ya existentes; reportándose en la literatura la implementación de Terapias Afirmativas con base teórica y/o técnica en diferentes tradiciones psicoterapéuticas tales como el psicoanálisis, la terapia sistémica o las terapias cognitivo-conductuales, entre otras.

Una vez explicitadas las variables generales de este marco referencial, Borges abordará en los restantes cinco capítulos una serie de temáticas específicas que permiten comprender en mayor profundidad el campo de aplicación y las características de los enfoques afirmativos. 
Así, en el capítulo 3, el autor analiza en profundidad el impacto de la homofobia - tanto social, como cultural, institucional e internalizada - y de la homoignoracia (Kort, 2008) en la atención psicológica a consultantes LGBTI.

Del mismo modo, en el capítulo 4, Borges se dedica a exponer los principales modelos teóricos vinculados a los procesos particulares de construcción y desarrollo de identidad LGBT (Borges, 2009, Capítulo 4), reflexionando además sobre su necesaria consideración en la práctica clínica concreta con la población referida; analizando en el capítulo 5 el impacto de estos elementos en la formación y desarrollo del terapeuta afirmativo.

Finalmente, el autor dedica los dos últimos capítulos a la reflexión sobre las particularidades de la clínica afirmativa. Para ello Borges analiza de forma desgranada en el capítulo 6 una serie de motivos de consulta frecuentes en dicha clínica, tales como los problemas de "armario", depresión, abuso de alcohol y drogas, sexualidad, relaciones afectivas, paternidad / maternidad, envejecimiento o infección por VIH / SIDA; destacando los elementos técnicos específicos a tener en cuenta en cada uno de estos casos.

Dicho análisis, junto con los elementos vertidos en los anteriores capítulos se ven fuertemente complementados por el capítulo 7, en el que el autor presenta y analiza viñetas y casos clínicos concretos.

El texto se cierra con el agregado de cuatro anexos que, sin duda alguna, constituyen apartado documental que contextualizan en mucho a los lectores, principalmente a quienes se aproximan por primera vez a este tipo de enfoques. Sea que se encuentren en posición de posible terapeuta, o se encuentren en posición de posibles consultantes 
Dichos anexos compilan:

1. La resolución del Consejo Federal de Psicología del Brasil $\mathrm{N} .{ }^{\circ} 001$ del 22 de marzo de 1999, en el cual se establecen normas de actuación en relación a la cuestión de la orientación sexual. Normas centradas en la no discriminación, la promoción y el bienestar de estas personas y de la humanidad como criterios éticos fundamentales y en el desarrollo específico de acciones tendientes a eliminar la discriminación y estigmatización de las prácticas homoeróticas y la eliminación de ejercicios profesionales que favorezcan la patologización y la coerción a tales prácticas.

2. Las directrices de la Asociación Americana de Psicología (APA) para la psicoterapia con clientes gays, lesbianas y bisexuales. Directrices actualizadas al día de hoy, con posterioridad a la edición del texto (American Psychological Association, 2012) y complementadas además con un instrumento similar para la atención de personas transgénero, de género fluido y no conformes con el género (American Psychological Association, 2015).

3. Orientaciones para encontrar un terapeuta afirmativo, cuya presentación es breve y se centra en las respuestas a preguntas como ¿qué es un terapeuta afirmativo gay? ¿por qué es tan importante para una persona LGBTI encontrar un terapeuta afirmativo?.

4. Un anexo con preguntas a realizarse por parte de los candidatos a terapeutas afirmativos, también breve, en el que se presentan algunas preguntas introspectivas tales como si el postulante considera que la orientación sexual puede o debe ser alterada, o si considera que una pareja homosexual puede establecer relaciones estables en el tiempo y satisfactorias. 


\section{Conclusiones}

Sin duda alguna, el aporte de Borges resulta indispensable y positivo por dos aspectos centrales.

En primer lugar, si bien existe literatura desarrollada desde enfoques afirmativos orientados a la presentación y análisis de situaciones concretas, como la desarrollada por Gabriel J. Martín (2016, 2017), el texto de Borges resulta ser el único publicado en iberoamérica donde se presenta el campo de las Psicologías Afirmativas de forma sistemática y desarrollada para un público profesional. Público que por barreras idiomáticas (Freitas De León, 2012) no ha dado cuenta de basar sus intervenciones con personas LGBTI en el ámbito regional.

En segundo lugar, porque ubica una posición profesional éticamente responsable, en medio de una coyuntura actual que resulta aún ambivalente. Coyuntura donde si bien se observan elementos transformadores positivos - acuñados en un largo recorrido de progreso y luchas por la igualdad social-, se registra también el surgimiento de novedosas situaciones regresivas y discriminatorias.

Situaciones tales como el reciente fallo judicial brasileño que ampara la práctica de las llamadas "terapias de reconversión" (Betim, 2017). Fallo que pasa por alto las recomendaciones y disposiciones éticas tanto del Consejo Federal de Psicología del Brasil - ya reseñada -, como de la Organización Panamericana de la Salud y la Organización Mundial de la Salud (OPS, 2012).

En virtud de todo lo señalado, el libro de Klecius Borges representa un baluarte de enorme importancia profesional tanto para las personas LGBTI que requieran prestaciones de atención psicológica - principalmente para comprender los contornos de la actuación profesional y su articulación con sus derechos civiles -, como para los profesionales que en diversos contextos deban realizar abordajes psicológicos con 
dicha población. Pero particularmente - y permítaseme enunciarlo - para aquellos poco pensados por la literatura que, como quien suscribe, compartimos la pertenencia simultánea a ambos grupos.

\section{Referencias}

American Psychological Association. (2015). Guidelines for Psychological Practice with Transgender and Gender Nonconforming People. American Psychologist, 70(9), 832-864. Recuperado de https://www.apa.org/practice/guidelines/transgender.pdf

American Psychological Association. (2012). Guidelines for psychological practice with lesbian, gay, and bisexual clients. American psychologist, 67(1), 10-42.

Ardila, R. (2007). Terapia afirmativa para homosexuales y lesbianas. Revista Colombiana de Psiquiatría, 36(1), 67-77.

Betim, F. (2017, septiembre 20). Indignación en Brasil después de que un juez autorice 'terapias' para gais. El País. Recuperado de https://elpais.com/internacional/2017/09/19/actualidad/1505853454_712122.ht $\mathrm{ml}$

Borges, K. (2009). Terapia afirmativa: uma introdução à psicologia e à psicoterapia dirigida a gays, lésbicas e bissexuais [Terapia afirmativa: una introducción a la psicología y a la psicoterapia dirigida a gays, lesbianas y bisexuales]. São Paulo: Edicoes GLS. Recuperado de https://www.amazon.es/TerapiaAfirmativa-Introdu\%C3\%A7\%C3\%A3o-Psicoterapia-psicoterapiaebook/dp/B00CF2W6US/ref=sr_1_1_twi_kin_1?s=digitaltext\&ie=UTF8\&qid=1499649053\&sr=1-1\&keywords=terapia + afirmativa 
Cass, V. C. (1979). Homosexuality identity formation: A theoretical model. Journal of homosexuality, 4(3), 219-235.

Coyle, A. E., \& Kitzinger, C. E. (2002). Lesbian and gay psychology: New perspectives. Oxford: Blackwell Publishing. Recuperado de http://psycnet.apa.org/psycinfo/2003-04616-000

Davies, D., \& Neal, C. (1996). Pink therapy: A guide for counsellors and therapists working with lesbian, gay, and bisexual clients (Vol. 1). Midenhead: McGrawHill Education.

Freitas De León, P. (2012). Entre "Agrado" e "Prudência", a identidade em questão: contribuições desde e para uma Psicologia Social Crítica no campo LGBT. En A. Lima (Ed.), Psicología Social Crítica: Paralaxes do contemporaneo. Porto Alegre: Editora Sulinas.

Kort, J. (2008). Gay affirmative therapy for the straight clinician: the essential guide. New York: W.W. Norton.

Martín, G. J. (2016). Quiérete mucho, maricón. Barcelona: Roca editorial.

Martín, G. J. (2017). El ciclo del amor marica. Barcelona: Roca editorial.

Neal, C., \& Davies, D. (Eds.). (2000). Issues in therapy with lesbian, gay, bisexual and transgender clients. Buckingham : Philadelphia : Open University Press.

OPS. (2012). OPS advierte que "terapias" de cambio de orientación sexual no tienen justificación médica y amenazan bienestar de personas. Washington: Pan American Health Organization - Organización Panamericana de la Salud. Recuperado de http://new.paho.org/hq/index.php?option=com_content\&task=view\&id=6803\&lte $\operatorname{mid}=1926$ 
Formato de citación

Freitas de León, P. (2018). Reseña: "Terapia afirmativa: una introducción a la psicología y a la psicoterapia dirigida a gays, lesbianas y bisexuales". Psicología, Conocimiento y Sociedad, 8(1) 288-298. doi: http://dx.doi.org/10.26864/PCS.v8.n1.13 